\title{
Maria Auxiliadora Hospital in Lima, Peru as a model for neurosurgical outreach to international charity hospitals
}

\author{
Melanie G. Hayden • Samuel Hughes • \\ Edward J. Hahn • Henry E. Aryan • Michael L. Levy • \\ Rahul Jandial
}

Received: 20 February 2010 /Accepted: 29 April 2010/Published online: 19 May 2010

(C) The Author(s) 2010. This article is published with open access at Springerlink.com

\begin{abstract}
Introduction A myriad of geopolitical and financial obstacles have kept modern neurosurgery from effectively reaching the citizens of the developing world. Targeted neurosurgical outreach by academic neurosurgeons to equip neurosurgical operating theaters and train local neurosurgeons is one method to efficiently and cost effectively improve sustainable care provided by international charity hospitals. The International Neurosurgical Children's As-
\end{abstract}

M. G. Hayden $(\bowtie)$

Department of Neurosurgery, Stanford University Medical Center, 300 Pasteur Road,

Stanford, CA 94305-5327, USA

e-mail: mghayden@gmail.com

S. Hughes

Division of Neurosurgery, Oregon Health Sciences University,

3303 SW Bond Ave,

Portland, CA 97239, USA

E. J. Hahn

Verdugo Hills Hospital,

1812 Verdugo Hills,

Glendale, CA 91208, USA

H. E. Aryan

Sierra Pacific Orthopaedic and Spine Center,

1630 E. Herndon Ave,

Fresno, CA 93720-3305, USA

M. L. Levy

Division of Pediatric Neurosurgery,

University of California at San Diego, Rady's Children's Hospital,

3020 Children's Way,

San Diego, CA 12923, USA

R. Jandial

City of Hope,

1500 East Duarte Road,

Duarte, CA 91010, USA sociation (INCA) effectively improved the available neurosurgical care in the Maria Auxiliadora Hospital of Lima, Peru through the advancement of local specialist education and training.

Methods Neurosurgical equipment and training were provided for the local neurosurgeons by a mission team from the University of California at San Diego.

Results At the end of 3 years, with one intensive week trip per year, the host neurosurgeons were proficiently and independently applying microsurgical techniques to previously performed operations, and performing newly learned operations such as neuroendoscopy and minimally invasive neurosurgery.

Conclusion Our experiences may serve as a successful template for the execution of other small scale, sustainable neurosurgery missions worldwide.

Keywords Endoscopic third ventriculostomy.

Neurosurgical training · Outreach

\section{Introduction}

Many regions of the developing world are still underserved in regard to access to neurosurgical care. Groups such as the Foundation for International Education in Neurosurgery (FIENS) have sought to bridge this gap. One effective and sustainable means of improving the medical care available is through the advancement of specialist education and training, in conjunction with providing the necessary surgical equipment [1,2].

The International Neurosurgical Children's Association (INCA) seeks, "to educate and equip those who dedicate their efforts to help the children most in need." INCA missions seek to provide neurosurgical renovation and 
enhancement by providing both equipment and training for standard and novel operations. Our mission to the Maria Auxiliadora Hospital of Lima, Peru enabled the host neurosurgeons to be proficient in microsurgical techniques and neuroendoscopy after only three intensive 1-week sessions. We also provided an operative microscope, microinstruments, endoscopy equipment, and a pneumatic drill. The experience of INCA in this setting may serve as a successful template for the construction of other small scale, sustainable neurosurgery missions worldwide.

\section{Methods}

All financial and administrative support were provided by Salah Hassenien-Variety Children's Lifeline, Solana Beach, California

Site identification and recruitment

A standard letter was drafted to present INCA's mission statement. This letter, directed to the host neurosurgeons and divisions, briefly introduced the goals and expectations of the program. This included disclosures, such as the lack of political or religious affiliations. Likewise, as INCA is dedicated to serving indigent children, the letter specified that these services were available to select international neurosurgical societies working within a charity hospital.

\section{Initial site evaluation}

Following this first contact, introductory information was obtained via phone calls and emails, detailing the host site's current resources, experience, needs, and expectations. Then an initial site evaluation visit was undertaken of selected sites that were most likely to successfully host and benefit from missions. Multiple categories of assessment of strengths were considered including skill level of faculty, facilities, political influence in hospital, and equipment. Each of these was scored on a scale of poor, average, good, and excellent. A separate column specified in detail the specific need or strength. The equipment evaluation was stratified into the assessment and recommendations for the operating room and ICU. Again the equipment was listed and graded with a detailed explanation. Equipment types evaluated included the operating table, Mayfield, pneumatic drill, microinstruments, microscope, endoscope, ultrasonic aspirator, bipolar coagulator, and ICU monitors. For each entry, a recommendation was included, describing either an intervention (including the corresponding cost), or no intervention. Images of the facility were taken for future reference as well as ultimate comparison when mission was completed.
Inaugural visit and subsequent visits

During the inaugural mission the visiting faculty were the primary surgeons, with the host team observing, assisting, and managing the operating room. The first 2 days were dedicated to running a preoperative clinic for case selection and scheduling. This was combined with relevant classroom instruction, to introduce new technology, and review the appropriate anatomical and surgical principles. These initial 2 days were also used to ensure functionality of the operative theater and equipment. The four operative days (days 3-6 of the mission) were scheduled with the more complicated cases first, in order to maximize time of postoperative observation. This first visit established the mission foundation and demonstrated the ability of the host team to successfully coordinate and execute the logistics of patient selection, operative schedule, and postoperative care, including obtaining hospital administrative support for anesthesia, nursing, and beds.

Having squared away the logistics in the first trip, the second mission was increasingly collaborative, focusing more on didactic elements. The host neurosurgeon and visiting faculty were paired for the duration of the trip. The host took an increasing role in operative cases and began to function as primary surgeon on some cases, in accordance with his level of skill.

On the third mission, the host neurosurgeon was primary on all the cases. By the end of the week, the visiting neurosurgeons continued in an observational role for even novel surgical techniques (such as neuroendoscopy). This final visit involved the mission team evaluation of host team performance in increasingly challenging specialty cases. In fact, many of the final cases were neuroendoscopy, microsurgical tumor, and vascular. This was made possible secondary to the one-on-one training in addition to the newly donated operative microscope and endoscope.

\section{Results}

The INCA mission to the Maria Auxiliadora Hospital in Lima, Peru, involved three visits, with travel and equipment costs totaling $\$ 40,000$. The skills and resources of the surgeons were best served through three missions to teach neuroendoscopy and microsurgical techniques. INCA provided the following refurbished or donated equipment: pneumatic drill, microinstruments, neuroendoscope, and operative microscope with observer scope. Two senior faculty were trained and currently function independently with basic neuroendoscopy. This was accomplished via 41 total cases split over three 1 -week visits per year for 3 years. Thirty-two of these cases directly involved host team participation. During the last visit, the host faculty utilized 
the endoscope not only for the treatment of hydrocephalus through a third ventriculostomy, but also for the country's first endoscopic transphenoidal and endoscopic assisted aneurysm clipping.

Most importantly, the number of endoscopy cases performed by the host team each year successively increased (Fig. 1). The host team continues to employ donated equipment to improve upon previously performed operations, as well as perform novel operations (in terms of premission expertise of host team), such as neuroendoscopy. Complications included one ventricular hemorrhage treated with 2 weeks of external ventricular drainage during the second visit and one meningitis during third visit.

\section{Discussion}

Modern neurosurgery has been slow to expand in developing countries secondary to the high cost of technology and the lack of modern neurosurgical training available to many local neurosurgeons [1, 3, 4]. Advancement of specialist education and training is an effective method for overall improvement of available medical care [1,2]. The idea is to encourage young local neurosurgeons to take responsibility as leaders of the specialty in their respective countries [1]. In order to excel, this should not be done in isolation, but rather incorporated into the local medical infrastructure [1]. Cooperative projects between developed and developing countries are effective means to create sustainable neurosurgery resources in underserved areas of the world [4-6].

Establishing a targeted and sustainable neurosurgery outreach programs from the developed to the developing world can effectively seek to minimize the ever-increasing gap between neurosurgical care. INCA missions are accomplished by the most effective use of mission resources with minimal commitment from American faculty. These missions create opportunities for providing new procedures and sustaining current treatment modalities. This passing of skill to the host team underscores the great potential for improving sustained neurosurgical care for the children of Lima, Peru. As part of the process, one must evaluate and accommodate different learning curves in order to expand the local armamentarium with minimal equipment.

\section{Initial site evaluation}

Before a mission can be constructed, a site evaluation visit must be undertaken. This may be done by one individual committed to the project, with experience in evaluating sites that are most compatible to the program. This 1-day visit allows physical inspection of the facilities and equipment, meeting with administrative head of the hospital, ICU, operating theaters, anesthesia as well as the host neurosurgery department chair.

Site evaluation and program selection seek the highest yield for indigent patients treated in government or charity hospitals. Following initial contact, the correct site selection is essential to a program's success. It is important to first understand the structure of the health care system, and envision a way the mission can work within these constraints. The site of the proposed visit must meet the standards for local physician commitment, patient population, nursing staff, ICU/post-op care, and follow up. Through discussion with this staff, one determines the patient population (regional and socioeconomic), case variety, case volume, and specific needs. The hospital with a demonstrable need for an intervention should be in an urban setting, preferable in a capital or major metropolitan city. It also should be a major referral center with an

\section{Endoscopic Cases}

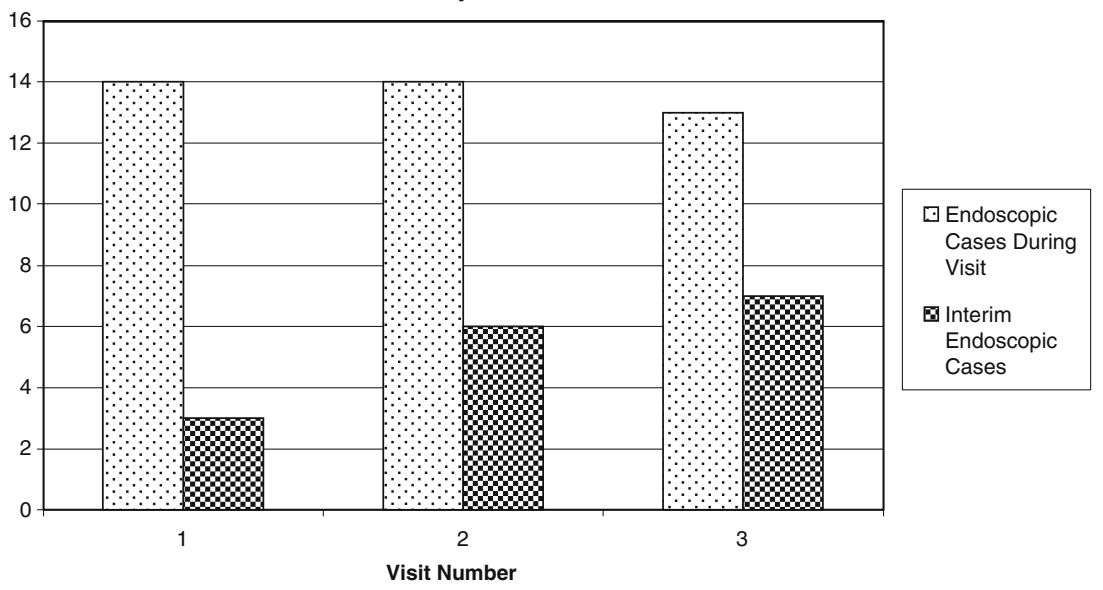

Fig. 1 Details of the number of endoscopic cases performed during the visit of the mission team, and the number of interim endoscopic cases performed by the host team 
academic department. The clinical facilities must be such that acceptable preoperative and postoperative resources exist. The host neurosurgeon must be dedicated, organized, and able to work with hospital administration and other medical/surgical services. It is important to keep in mind that the perceived educational needs of the local surgeon may differ between the host site and visiting medical team, reflecting the differences in training environments and medical culture [7].

At this site selection visit a specific intervention may be determined, utilizing the strengths of the site and understanding the limitations. A projected outcome of the intervention may also be estimated. The decision must then be made whether to plan and carry out a mission where appropriate.

In the case of the Maria Auxiliadora Hospital, neuroendoscopic treatment of hydrocephalus was thought to potentially reduce shunting by $20-30 \%$ in the children served. Likewise, the academic center needed a microscope for microsurgical technique and an endoscope, for patient care as well as for teaching neurosurgical residents.

\section{Inaugural visit and subsequent visits}

Following the initial site evaluation, previsit preparations must begin. This includes media outreach, creating a list of potential patients, and arranging customs issues for equipment transfer. In addition, a medical team is constructed, keeping numbers low to limit costs and maximize efficiency. The attending neurosurgeon should be proficient and comfortable teaching the area of expertise to be exported. Ideally, the host site will have scheduled a 2-day clinic followed by four dedicated operative days. The inaugural mission serves primarily to judge the host team's ability to coordinate and bypass the obstacles in place to hosting a 1-week mission. Accordingly, while evaluating preparedness of operative ancillary staff and other critical elements, the inaugural mission focuses on cases that are not likely to incur many operative or postoperative complications. Once confidence is gained about the efficacy of hosting a mission, the subsequent missions can escalate the level of complicated cases. The first visit establishes the foundation from which the subsequent mission can serve as didactic missions for the host neurosurgeons.

The medical missions lead to increased international collaboration between the mission and host teams and hospitals. Specifically, the host site benefits from new technology and training provided and perpetuates this to their citizens when the targeted missions conclude. As seen by the proficiency of the host neurosurgeons at the end of the third mission, the combination of equipment and training ensures sustainability.

\section{Conclusion}

The purpose of targeted neurosurgery outreach is to create sustainable programs serving indigent patients in an area with demonstrated need yet sufficient infrastructure to nourish the new resources and surgical skills. To this end, one must focus on high function rather than high tech and maintain the host team capacity to be resourceful. Maria Auxiliadora serves as a model for what three 1-week collaborative missions can accomplish. The final visit demonstrated that with international neurosurgical collaboration, our efforts can resonate profoundly when working with the dedicated and resourceful host surgeons in many nations worldwide.

Acknowledgments The missions described above would not have been possible without the generous support of Salah Hassenien and Variety Children's Lifeline.

Open Access This article is distributed under the terms of the Creative Commons Attribution Noncommercial License which permits any noncommercial use, distribution, and reproduction in any medium, provided the original author(s) and source are credited.

\section{References}

1. El Khamlichi A (1999) Technology and neurosurgery in developing countries: experience and present situation in Morocco. Neurosurgery 454:896-900

2. Lee ST (2001) International education: 20 years after implementation of a training program. Neurosurgery 486:1348-1350, discussion $1350-1351$

3. Ramamurthi B (2004) Appropriate technology for neurosurgery. Surg Neurol 612:109-112, discussion 112-116

4. Warf BC (2005) Comparison of endoscopic third ventriculostomy alone and combined with choroid plexus cauterization in infants younger than 1 year of age: a prospective study in 550 African children. J Neurosurg 1036(Suppl):475-481

5. Mukhida K, Shilpakar SK, Sharma MR, Bagan M (2005) Neurosurgery at Tribhuvan University Teaching Hospital, Nepal. Neurosurgery 571:172-180, discussion $172-180$

6. Rosenfeld JV, Xuan NT (1997) Neurosurgery in Vietnam. Surg Neurol 483:307-311

7. Bernstein M, Hamstra SJ, Woodrow S, Goldsman S, Reznick RK, Fairholm D (2006) Needs assessment of neurosurgery trainees: a survey study of two large training programs in the developing and developed worlds. Surg Neurol 662:117-124, discussion 124-126 